\title{
ON INFINITELY DIFFERENTIABLE AND GEVREY VECTORS FOR REPRESENTATION
}

\author{
A.F.M. TER ELST
}

(Communicated by Palle E. T. Jorgensen)

\begin{abstract}
In the present paper we give a condition in order that the set of infinitely differentiable vectors for a representation $\pi$ in a Banach space be equal to the set of all infinitely differentiable vectors for the restriction of $\pi$ to a subgroup. Similar results for Gevrey vectors and analytic vectors are proved for unitary representations.
\end{abstract}

\section{INTRODUCTION AND NOTATIONS}

Let $\pi$ be a continuous representation of a $d$-dimensional real Lie group $G$ in a Banach space $E$. For each $u \in E$, define $\tilde{u}: G \rightarrow E$ by $\tilde{u}(x):=\pi_{x} u(x \in G)$. A vector $u \in E$ is said to be infinitely differentiable, analytic resp. a Gevrey vector of order $\lambda$ for $\pi, \lambda \geq 1$, if the map $\tilde{u}$ is infinitely differentiable, (real) analytic resp. a Gevrey function of order $\lambda$ for $\pi$. (Cf. [Går], [Ne1], and [GW], respectively.) Let $D^{\infty}(\pi), D^{\omega}(\pi)$, and $G_{\lambda}(\pi)$ denote the space of all infinitely differentiable vectors, of all analytic vectors, and of all Gevrey vectors of order $\lambda$ for $\pi$, respectively. Note that $D^{\omega}(\pi)=G_{1}(\pi)$. For each $X$ in the Lie algebra $\mathfrak{g}$ of $G$, let $d \pi(X)$ denote the infinitesimal generator of the oneparameter group $t \mapsto \pi_{\exp t X}$ and let $\partial \pi(X)$ denote the restriction of $d \pi(X)$ to $D^{\infty}(\pi)$. The map $X \mapsto \partial \pi(X)$ extends uniquely to an associative algebra homomorphism from the complex universal enveloping algebra $U(\mathfrak{g})$ of $\mathfrak{g}$ into the set of all linear operators from $D^{\infty}(\pi)$ into $D^{\infty}(\pi)$. The extension is denoted by $\partial \pi$ also.

There exist infinitesimal characterizations for the spaces $D^{\infty}(\pi), D^{\omega}(\pi)$, and $G_{\lambda}(\pi)$. Therefore, let $\mathscr{A}$ be a set of (possibly unbounded) operators in $E$. Define the joint $C^{\infty}$-domain $D^{\infty}(\mathscr{A})$ of the set $\mathscr{A}$ by

$$
D^{\infty}(\mathscr{A}):=\bigcap_{n \in \mathbb{N}_{0}} \bigcap_{A_{1}, \ldots, A_{n} \in \mathscr{A}} D\left(A_{1} \circ \cdots \circ A_{n}\right) .
$$

Here $D\left(A_{1} \circ \cdots \circ A_{n}\right)$ denotes the domain of the operator $A_{1} \circ \cdots \circ A_{n}$. For

Received by the editors January 15, 1990.

1980 Mathematics Subject Classification (1985 Revision). Primary 47D30, 22E46, 22 E45. 
$\lambda \geq 1$, define the Gevrey space $S_{\lambda}(\mathscr{A})$ of order $\lambda$ relative to $\mathscr{A}$ by

$$
S_{\lambda}(\mathscr{A}):=\left\{u \in D^{\infty}(\mathscr{A}): \exists_{c, t>0} \forall_{n \in \mathbb{N}_{0}} \forall_{A_{1}, \ldots, A_{n} \in \mathscr{A}}\left[\left\|A_{1} \circ \cdots \circ A_{n} u\right\| \leq c t^{n} n !^{\lambda}\right]\right\} .
$$

(Cf. [GW, §1].) Now Goodman and Wallach have proved the following infinitesimal characterization of the spaces $D^{\infty}(\pi)$ and $G_{\lambda}(\pi)$ :

Theorem 1. Let $\pi$ be a representation of a Lie group $G$ in a Banach space $E$. Let $X_{1}, \ldots, X_{d}$ be any basis in the Lie algebra $\mathfrak{g}$ of $G$. Let $\lambda \geq 1$. Then

$$
D^{\infty}(\pi)=D^{\infty}\left(\left\{d \pi\left(X_{1}\right), \ldots, d \pi\left(X_{d}\right)\right\}\right)
$$

and

$$
G_{\lambda}(\pi)=S_{\lambda}\left(\left\{d \pi\left(X_{1}\right), \ldots, d \pi\left(X_{d}\right)\right\}\right) .
$$

Proof. See [Goo, Proposition 1.1] and [GW, Proposition 1.5].

Let $d_{1} \in\{1, \ldots, d-1\}$, where $d=\operatorname{dim} \mathfrak{g}$. Then clearly, for any basis $X_{1}, \ldots, X_{d}$ in $\mathfrak{g}$ :

$$
D^{\infty}(\pi)=D^{\infty}\left(\left\{d \pi\left(X_{1}\right), \ldots, d \pi\left(X_{d}\right)\right\}\right) \subset D^{\infty}\left(\left\{d \pi\left(X_{1}\right), \ldots, d \pi\left(X_{d_{1}}\right)\right\}\right) .
$$

In the present paper, we give conditions on the Lie algebra $\mathfrak{g}$ and the representation $\pi$ in order that $D^{\infty}(\pi)=D^{\infty}\left(\left\{d \pi\left(X_{1}\right), \ldots,\left(X_{d_{1}}\right)\right\}\right)$ for suitable $X_{1}, \ldots, X_{d_{1}}$ in $\mathfrak{g}$. Also, in case $\mathfrak{k}:=\operatorname{span}\left\{d \pi\left(X_{1}\right), \ldots, d \pi\left(X_{d_{1}}\right)\right\}$ is a subalgebra of $\mathfrak{g}$, there exists a subgroup $K$ of $G$ with Lie algebra $\mathfrak{k}$ and we obtain

$$
D^{\infty}(\pi)=D^{\infty}\left(\left.\pi\right|_{K}\right)
$$

For a semisimple Lie group $G$, these conditions are satisfied if for $\mathfrak{k}$ we take the subalgebra $\mathfrak{k}$ in the Cartan decomposition $\mathfrak{g}=\mathfrak{k}+\mathfrak{p}$ of $\mathfrak{g}$, and for the representation $\pi$ we take a completely irreducible one or a principal series representation.

For unitary representations we prove similar theorems for the set of Gevrey vectors.

\section{INFINITELY DIFFERENTIABLE VECTORS FOR RESTRICTIONS TO SUBGROUPS}

In this section we prove the following theorem:

Theorem 2. Let $\pi$ be a representation of a Lie group $G$ in a Banach space $E$. Let $X_{1}, \ldots, X_{d}$ be a basis in the Lie algebra $\mathfrak{g}$ of $G$. Let $d_{1} \in\{1, \ldots, d-1\}$, and let

$$
C:=X_{1}^{2}+\cdots+X_{d_{1}}^{2}-X_{d_{1}+1}^{2}-\cdots-X_{d}^{2} \in U(\mathfrak{g}) .
$$


Suppose $C$ belongs to the center of $U(\mathfrak{g})$, and suppose there exists $\tau \in \mathbb{C}$ such that

$$
\partial \pi(C)=\tau I
$$

Then

$$
D^{\infty}(\pi)=D^{\infty}\left(\left\{d \pi\left(X_{1}\right), \ldots, d \pi\left(X_{d}\right)\right\}\right)=D^{\infty}\left(\left\{d \pi\left(X_{1}\right), \ldots, d \pi\left(X_{d_{1}}\right)\right\}\right) .
$$

Proof. Without loss of generality, we may assume that $G$ is connected. Let

$$
\begin{aligned}
\Delta & :=X_{1}^{2}+\cdots+X_{d}^{2} \in U(\mathfrak{g}), \\
\Delta_{1} & :=X_{1}^{2}+\cdots+X_{d_{1}}^{2} \in U(\mathfrak{g}), \\
\tilde{\Delta} & :=\widetilde{X}_{1}^{2}+\cdots+\widetilde{X}_{d}^{2}, \\
\tilde{\Delta}_{1} & :=\widetilde{X}_{1}^{2}+\cdots+\widetilde{X}_{d_{1}}^{2} .
\end{aligned}
$$

Here $\tilde{X}$ denotes the left invariant differential operator on $G$, which corresponds to $X$. Let $u \in D^{\infty}\left(\left\{d \pi\left(X_{1}\right), \ldots, d \pi\left(X_{d_{1}}\right)\right\}\right)$ be fixed. We have to prove that the function $\tilde{u}$ from $G$ into $E$ is infinitely differentiable. By [Pou], it is enough to prove that $\tilde{u}$ is weakly infinitely differentiable; i.e., the function $f \circ \tilde{u}$ from $G$ into $\mathbb{C}$ is infinitely differentiable for all $f \in E^{\prime}$. We shall show that, for all $f \in E^{\prime}$ and all $m \in \mathbb{N}$, there exists a continuous function $g$ on $G$ such that $f \circ \tilde{u}$ is a weak solution of the equation $\widetilde{\Delta}^{m} F=g$; then, by using regularity theory for elliptic differential operators, the regularity of $f \circ \tilde{u}$ follows.

Let $\check{\pi}$ be the contragredient representation of $\pi$ on the Banach space $\check{E}$ in the sense of Bruhat. So $\check{E}$ consists of all $f \in E^{\prime}$ for which the map $x \mapsto$ $\left(\pi_{x^{-1}}\right)^{*} f$ from $G$ into $E^{\prime}$ is (strongly) continuous. (Here ()$^{*}$ denotes the dual operator in the dual space.) Then, for all $x \in G$, the operator $\check{\pi}_{x}$ is defined by $\check{\pi}_{x}:=\left.\left(\pi_{x^{-1}}\right)^{*}\right|_{\check{E}}$. So $x \mapsto \check{\pi}_{x}$ is a continuous representation of $G$ in the Banach space $\check{E}$. (See [Bru, $\S I .2 .2]$.) We first consider infinitely differentiable vectors of $\check{\pi}$. Let $f \in D^{\infty}(\check{\pi})$. Then $f \circ \tilde{u}(x)=f\left(\pi_{x} u\right)=\left[\check{\pi}_{x^{-1}} f\right](u)$ for all $x \in G$, so $f \circ \tilde{u}$ is an infinitely differentiable function from $G$ into $\mathbb{C}$. Let $m \in \mathbb{N}$. Let

$$
w_{m}:=\left(2 \sum_{k=1}^{d_{1}}\left[d \pi\left(X_{k}\right)\right]^{2}-\tau I\right)^{m} u .
$$

Recall that $u \in D^{\infty}\left(\left\{d \pi\left(X_{1}\right), \ldots, d \pi\left(X_{d_{1}}\right)\right\}\right)$.

Assertion 1. For all $f \in D^{\infty}(\check{\pi})$ and all $x \in G$, we have

$$
\left[\tilde{\Delta}^{m}(f \circ \tilde{u})\right](x)=\left[f \circ \widetilde{w}_{m}\right](x) .
$$


Proof of Assertion 1. Let $f \in D^{\infty}(\check{\pi})$. Let $n \in \mathbb{N}$ and let $j_{1}, \ldots, j_{n} \in$ $\{1, \ldots, d\}$. Then, for all $x \in G$ :

$$
\begin{aligned}
{\left[\tilde{X}_{j_{1}} \circ \cdots \circ \tilde{X}_{j_{n}}(f \circ \tilde{u})\right](x) } \\
\quad=\left.\left.\frac{\partial}{\partial t_{1}}\right|_{0} \cdots \frac{\partial}{\partial t_{n}}\right|_{0} f\left(\pi_{x} \pi_{\exp \left(t_{1} X_{j_{1}}\right)} \circ \cdots \circ \pi_{\exp \left(t_{n} X_{j_{n}}\right)} u\right) \\
\quad=\left.\left.\frac{\partial}{\partial t_{1}}\right|_{0} \cdots \frac{\partial}{\partial t_{n}}\right|_{0} f\left(\pi_{\exp \left(t_{1} \operatorname{Ad}(x) X_{j_{1}}\right)} \circ \cdots \circ \pi_{\left.\exp \left(t_{n} \operatorname{Ad}(x) X_{j_{n}}\right) \pi_{x} u\right)}\right. \\
\quad=\left.\left.\frac{\partial}{\partial t_{1}}\right|_{0} \cdots \frac{\partial}{\partial t_{n}}\right|_{0}\left[\check{\pi}_{\exp \left(-t_{n} \operatorname{Ad}(x) X_{j_{n}}\right)} \circ \cdots \circ \check{\pi}_{\exp \left(-t_{1} \operatorname{Ad}(x) X_{j_{1}}\right)} f\right]\left(\pi_{x} u\right) \\
\quad=(-1)^{n}\left[\partial \check{\pi}\left(\operatorname{Ad}(x)\left(X_{j_{n}} \cdots X_{j_{1}}\right)\right) f\right]\left(\pi_{x} u\right) \\
\quad=\left[\partial \check{\pi}\left(\operatorname{Ad}(x)\left(X_{j_{1}} \cdots X_{j_{n}}\right)^{*}\right) f\right]\left(\pi_{x} u\right) .
\end{aligned}
$$

Here $L \mapsto L^{*}$ denotes the usual antiautomorphism from $U(\mathfrak{g})$ onto $U(\mathfrak{g})$. Let $Y \in \mathfrak{g}$. Then $\operatorname{Ad}(\exp Y)(C)=e^{\operatorname{ad} Y}(C)=C$, because $C$ belongs to the center of $U(\mathfrak{g})$. Since $G$ is connected, $\operatorname{Ad}(x)(C)=C$ for all $x \in G$. Moreover, for all $v \in D^{\infty}(\pi)$, we have

$$
[\partial \check{\pi}(C) f](v)=f\left(\partial \pi\left(C^{*}\right) v\right)=f(\partial \pi(C) v)=\tau f(v) .
$$

Since $D^{\infty}(\pi)$ is dense in $E$, by [Går], $\partial \check{\pi}(C) f=\tau f$, by continuity. Note that $\Delta=2 \Delta_{1}-C$. So we obtain for all $x \in G$ :

$$
\begin{aligned}
{\left[\tilde{\Delta}^{m}(f \circ \tilde{u})\right](x) } & =\left[\partial \check{\pi}\left(\operatorname{Ad}(x)\left(\Delta^{m}\right)\right) f\right]\left(\pi_{x} u\right) \\
& =\sum_{k=0}^{m}(-1)^{k}\left(\begin{array}{c}
m \\
k
\end{array}\right)\left[\{\partial \check{\pi}(\operatorname{Ad}(x)(C))\}^{k} \partial \check{\pi}\left(\operatorname{Ad}(x)\left(2 \Delta_{1}\right)^{m-k}\right) f\right]\left(\pi_{x} u\right) \\
& =\sum_{k=0}^{m}(-1)^{k}\left(\begin{array}{c}
m \\
k
\end{array}\right)\left[\tau^{k} \partial \check{\pi}\left(\operatorname{Ad}(x)\left(2 \Delta_{1}\right)^{m-k}\right) f\right]\left(\pi_{x} u\right) \\
& =\left[\partial \check{\pi}\left(\operatorname{Ad}(x)\left(\left(2 \Delta_{1}-\tau\right)^{m}\right)\right) f\right]\left(\pi_{x} u\right) \\
& =\left[\left(2\left(\widetilde{\Delta}_{1}-\tau\right)^{m}(f \circ \tilde{u})\right](x)\right. \\
& =\left[\left(f \circ \widetilde{w}_{m}\right)\right](x) .
\end{aligned}
$$

This proves Assertion 1.

Let $\lambda$ be a right Haar measure on $G$.

Assertion 2. For all $\varphi \in C_{c}^{\infty}(G)$ and all $f \in E^{\prime}$, we have

$$
\int_{G}\left[\tilde{\Delta}^{m} \varphi\right](x)[f \circ \tilde{u}](x) d \lambda(x)=\int_{G} \varphi(x)\left[f \circ \widetilde{w}_{m}\right](x) d \lambda(x)
$$

Proof of Assertion 2. Let $T_{K}$ be the polar topology for $E^{\prime}$ of uniform convergence on the compact subsets of $E$. Since $E$ is complete, the topology $T_{K}$ is compatible with the dual pair $\left(E^{\prime}, E\right)$, by [Wil, Example 9-2-10 and Theorem 9-2-12]. Now it follows by the same arguments as in [Bru, p. 113] that $\check{E}$ is not only $w^{*}$-dense in $E^{\prime}$, but even dense in $\left(E^{\prime}, T_{K}\right)$. 
Since $D^{\infty}(\check{\pi})$ is dense in the Banach space $\check{E}$ and since $\check{E}$ is dense in $\left(E^{\prime}, T_{K}\right)$, it follows that $D^{\infty}(\check{\pi})$ is dense in $\left(E^{\prime}, T_{K}\right)$. Now let $f \in E^{\prime}$ and $\varphi \in C_{c}^{\infty}(G)$. Let $\varepsilon>0$, let $K:=\left\{\pi_{x} u: x \in \operatorname{supp} \varphi\right\} \cup\left\{\pi_{x} w_{m}: x \in \operatorname{supp} \varphi\right\}$ and $M:=1+\int_{G}\left|\widetilde{\Delta}^{m} \varphi(x)\right| d \lambda(x)+\int_{G}|\varphi(x)| d \lambda(x)$. There exists $g \in D^{\infty}(\check{\pi})$ such that for all $a \in K:|f(a)-g(a)| \leq \varepsilon M^{-1}$. Then, by Assertion 1,

$$
\begin{aligned}
\int_{G}\left[\tilde{\Delta}^{m} \varphi\right](x)[g \circ \tilde{u}](x) d \lambda(x) & =\int_{G} \varphi(x)\left[\tilde{\Delta}^{m}(g \circ \tilde{u})\right](x) d \lambda(x) \\
& =\int_{G} \varphi(x)\left[g \circ \widetilde{w}_{m}\right](x) d \lambda(x) .
\end{aligned}
$$

So

$$
\begin{aligned}
\left|\int_{G}\left[\tilde{\Delta}^{m} \varphi\right](x)[f \circ \tilde{u}](x) d \lambda(x)-\int_{G} \varphi(x)\left[f \circ \widetilde{w}_{m}\right](x) d \lambda(x)\right| \\
\leq \int_{G}\left|\left[\tilde{\Delta}^{m} \varphi\right](x)\left(f\left(\pi_{x} u\right)-g\left(\pi_{x} u\right)\right)\right| d \lambda(x) \\
\quad+\left|\int_{G}\left[\tilde{\Delta}^{m} \varphi\right](x)[g \circ \tilde{u}](x) d \lambda(x)-\int_{G} \varphi(x)\left[g \circ \widetilde{w}_{m}\right](x) d \lambda(x)\right| \\
\quad+\int_{G}\left|\varphi(x)\left(g\left(\pi_{x} w_{m}\right)-f\left(\pi_{x} w_{m}\right)\right)\right| d \lambda(x) \\
\leq \varepsilon M^{-1}\left(\int_{G}\left|\widetilde{\Delta}^{m} \varphi(x)\right| d \lambda(x)+\int_{G}|\varphi(x)| d \lambda(x)\right) \\
\leq \varepsilon .
\end{aligned}
$$

This proves Assertion 2.

Now we prove the theorem. Let $f \in E^{\prime}$. By Assertion 2, the function $f \circ \tilde{u}$ is a weak solution of the equation $\widetilde{\Delta}^{m} F=f \circ \widetilde{w}_{m}$. Since $f \circ \widetilde{w}_{m}$ is a continuous function and $\widetilde{\Delta}^{m}$ is an elliptic operator of order $2 m$, it follows from the local regularity theorem for elliptic operators that $f \circ \tilde{u}$ has locally $L^{2}$ derivatives of order $\leq 2 m$. (See [Fol, Theorem 6.30].) Hence by [Fol, Lemma 6.9] (the Sobolev lemma), the function $f \circ \tilde{u}$ is $2 m-d$ times continuously differentiable. Therefore $f \circ \tilde{u}$ is infinitely differentiable for all $f \in E^{\prime}$ and hence the function $\tilde{u}$ is infinitely differentiable. Thus $u \in D^{\infty}(\pi)$.

Corollary 3. Let $G$ be a semisimple Lie group with Lie algebra $\mathfrak{g}$. Let $\pi$ be a representation of $G$ in a Banach space. Let $C \in U(\mathfrak{g})$ be the Casimir element. Suppose there exists $\tau \in \mathbb{C}$ such that $\partial \pi(C)=\tau I$. Let $\mathfrak{g}=\mathfrak{k}+\mathfrak{p}$ be a Cartan decomposition of $\mathfrak{g}$, and let $K$ be a subgroup of $G$ with Lie algebra $\mathfrak{k}$. Then

$$
D^{\infty}(\pi)=D^{\infty}\left(\left.\pi\right|_{K}\right)
$$

Proof. Let $B$ denote the Killing form of $\mathfrak{g}$. Let $X_{1}, \ldots, X_{d_{1}}$ be a basis in $\mathfrak{k}$ and $X_{d_{1}+1}, \ldots, X_{d}$ be a basis in $\mathfrak{p}$ such that $B\left(X_{i}, X_{j}\right) \stackrel{d_{d, j}}{=}-\delta_{i, j}$ for all $1 \leq i, j \leq d_{1}$ and $B\left(X_{i}, X_{j}\right)=\delta_{i, j}$ for all $d_{1}<i, j \leq d$. Then $C=$ $\sum_{k=d_{1}+1}^{d} X_{k}^{2}-\sum_{k=1}^{d_{1}} X_{k}^{2}$. So, by Theorems 2 and 1, we obtain that

$$
D^{\infty}(\pi)=D^{\infty}\left(\left\{d \pi\left(X_{1}\right), \ldots, d \pi\left(X_{d_{1}}\right)\right\}\right)=D^{\infty}\left(\left.\pi\right|_{K}\right) .
$$


Remark. Note that there are no conditions on the center of $G$ in the previous corollary.

Corollary 4. Let $G$ be a connected semisimple Lie group with finite center. Let $K$ be a maximal compact subgroup. Let $\pi$ be a principal series representation of $G$. Then $D^{\infty}(\pi)=D^{\infty}\left(\left.\pi\right|_{K}\right)$.

Corollary 5. Let $\pi$ be a completely irreducible representation of a Lie group $G$ in a Banach space. Let $X_{1}, \ldots, X_{d}$ be a basis in the Lie algebra $\mathfrak{g}$ of $G$. Let $d_{1} \in\{1, \ldots, d-1\}$. Let

$$
C:=X_{1}^{2}+\cdots+X_{d_{1}}^{2}-X_{d_{1}+1}^{2}-\cdots-X_{d}^{2} \in U(\mathfrak{g}) .
$$

Suppose $C$ belongs to the center of $U(\mathfrak{g})$. Then

$$
D^{\infty}(\pi)=D^{\infty}\left(\left\{d \pi\left(X_{1}\right), \ldots, d \pi\left(X_{d_{1}}\right)\right\}\right) .
$$

Proof. Since $\pi$ is completely irreducible, by [Tay, Proposition 0.4.5], there exists $\tau \in \mathbb{C}$ such that $\partial \pi(C)=\tau I$.

\section{GEVREY VECTORS FOR RESTRICTIONS TO SUBGROUPS}

In this section we prove a theorem similar to the results of the previous section, but now for Gevrey vectors instead of infinitely differentiable vectors. However, in this section we consider only unitary representations. We immediately formulate the main theorem of this section:

Theorem 6. Let $\pi$ be a unitary representation of $G$. Let $X_{1}, \ldots, X_{d}$ be a basis in the Lie algebra $\mathfrak{g}$ of $G$. Let $d_{1} \in\{1, \ldots, d-1\}$. Let

$$
C:=X_{1}^{2}+\cdots+X_{d_{1}}^{2}-X_{d_{1}+1}^{2}-\cdots-X_{d}^{2} \in U(\mathfrak{g}) .
$$

Suppose $C$ belongs to the center of $U(\mathfrak{g})$, and suppose there exists $\tau \in \mathbb{R}$ such that

$$
\partial \pi(C)=\tau I .
$$

Let $\lambda \geq 1$. Then

$$
G_{\lambda}(\pi)=S_{\lambda}\left(\left\{d \pi\left(X_{1}\right), \ldots, d \pi\left(X_{d}\right)\right\}\right)=S_{\lambda}\left(\left\{d \pi\left(X_{1}\right), \ldots, d \pi\left(X_{d_{1}}\right)\right\}\right) .
$$

In particular,

$$
D^{\omega}(\pi)=S_{1}\left(\left\{d \pi\left(X_{1}\right), \ldots, d \pi\left(X_{d_{1}}\right)\right\}\right) .
$$

Proof. First we prove that

$$
S_{\lambda}\left(\left\{\partial \pi\left(X_{1}\right), \ldots, \partial \pi\left(X_{d}\right)\right\}\right)=S_{\lambda}\left(\left\{\partial \pi\left(X_{1}\right), \ldots, \partial \pi\left(X_{d_{1}}\right)\right\}\right) .
$$

Let $\Delta:=X_{1}^{2}+\cdots+X_{d}^{2} \in \in^{\prime} U(\mathfrak{g})$ and $\Delta_{1}:=X_{1}^{2}+\cdots+X_{d_{1}}^{2} \in U(\mathfrak{g})$ be as in the proof of Theorem 2. Let $u \in S_{\lambda}\left(\left\{\partial \pi\left(X_{1}\right), \ldots, \partial \pi\left(X_{d_{1}}\right)\right\}\right)$. By an elementary counting argument it easily follows that $u \in S_{2 \lambda}\left(\left\{\partial \pi\left(\Delta_{1}\right)\right\}\right)$. Let $C, t>0$ be such that

$$
\left\|\left[\partial \pi\left(\Delta_{1}\right)\right]^{n} u\right\| \leq C t^{n} n !^{2 \lambda}
$$


for all $n \in \mathbb{N}_{0}$. Since $\partial \pi(\Delta)=2 \partial \pi\left(\Delta_{1}\right)-\partial \pi(C)=2 \partial \pi\left(\Delta_{1}\right)-\tau I$, we obtain for all $n \in \mathbb{N}_{0}$

$$
\begin{aligned}
\left\|[\partial \pi(\Delta)]^{n} u\right\| & \leq \sum_{k=0}^{n}\left(\begin{array}{l}
n \\
k
\end{array}\right) 2^{k}|\tau|^{n-k}\left\|\left[\partial \pi\left(\Delta_{1}\right)\right]^{k} u\right\| \\
& \leq C \sum_{k=0}^{n}\left(\begin{array}{l}
n \\
k
\end{array}\right) 2^{k} t^{k}|\tau|^{n-k} k !^{2 \lambda} \\
& \leq C n !^{2 \lambda} \sum_{k=0}^{n}\left(\begin{array}{l}
n \\
k
\end{array}\right) 2^{k} t^{k}|\tau|^{n-k} \\
& =C(2 t+|\tau|)^{n} n !^{2 \lambda} .
\end{aligned}
$$

So $u \in S_{2 \lambda}(\{\partial \pi(\Delta)\})$.

Now by [GW, example following Theorem 1.7], we obtain that $u \in$ $S_{\lambda}\left(\left\{\partial \pi\left(X_{1}\right), \ldots, \partial \pi\left(X_{d}\right)\right\}\right)$. (Here we used the fact that $\pi$ is a unitary representation.) So

$$
S_{\lambda}\left(\left\{\partial \pi\left(X_{1}\right), \ldots, \partial \pi\left(X_{d_{1}}\right)\right\}\right) \subset S_{\lambda}\left(\left\{\partial \pi\left(X_{1}\right), \ldots, \partial \pi\left(X_{d}\right)\right\}\right) .
$$

Thus

$$
S_{\lambda}\left(\left\{\partial \pi\left(X_{1}\right), \ldots, \partial \pi\left(X_{d_{1}}\right)\right\}\right)=S_{\lambda}\left(\left\{\partial \pi\left(X_{1}\right), \ldots, \partial \pi\left(X_{d}\right)\right\}\right) .
$$

By Theorem 2 we have the equality of the joint $C^{\infty}$-domains

$$
D^{\infty}\left(\left\{d \pi\left(X_{1}\right), \ldots, d \pi\left(X_{d_{1}}\right)\right\}\right)=D^{\infty}\left(\left\{d \pi\left(X_{1}\right), \ldots, d \pi\left(X_{d}\right)\right\}\right) .
$$

So

$$
S_{\lambda}\left(\left\{d \pi\left(X_{1}\right), \ldots, d \pi\left(X_{d_{1}}\right)\right\}\right)=S_{\lambda}\left(\left\{d \pi\left(X_{1}\right), \ldots, d \pi\left(X_{d}\right)\right\}\right) .
$$

This proves the theorem.

Remark. Another proof of this theorem has been presented in [tE, p. 102].

Now, for the Gevrey vectors for unitary representations, we can state the same type of corollaries as in $\S 2$, for example the following:

Corollary 7. Let $G$ be a semisimple Lie group with Lie algebra $\mathfrak{g}$. Let $\pi$ be a unitary representation of $G$. Let $C \in U(\mathfrak{g})$ be the Casimir element. Suppose there exists $\tau \in \mathbb{C}$ such that $\partial \pi(C)=\tau I$. (For example, $\pi$ is irreducible.) Let $\mathfrak{g}=\mathfrak{k}+\mathfrak{p}$ be a Cartan decomposition of $\mathfrak{g}$, and let $K$ be a subgroup of $G$ with Lie algebra $\mathfrak{k}$. Then

$$
G_{\lambda}(\pi)=G_{\lambda}\left(\left.\pi\right|_{K}\right)
$$

In particular,

$$
D^{\omega}(\pi)=D^{\omega}(\pi \mid K)
$$

Added in proof. Since the paper was submitted, D. W. Robinson pointed out to the author that the results in $\S 3$ are also valid for Banach space representa- 
tions, since $\left.G_{\lambda}(\pi)=S_{2 \lambda}(\{\partial \pi(\Delta))\}\right)$. (See [Rob, p. 390 and Theorem 7.1], with obvious modifications for the case $\lambda>1$.)

\section{ACKNOWLEDGMENT}

The author wishes to thank G. van Dijk, S. J. L. van Eijndhoven, and J. de Graaf for their suggestions and comments.

\section{REFERENCES}

[Bru] F. Bruhat, Sur les représentations induites des groupes de Lie, Bull. Soc. Math. France 84 (1956), 97-205.

[tE] A.F.M. ter Elst, Gevrey spaces related to Lie algebras of operators, Thesis, Eindhoven University of Technology, Eindhoven, The Netherlands, 1989.

[Fol] G. B. Folland, Introduction to partial differential equations, Princeton University Press, Princeton, NJ, 1976.

[Gảr] L. Gảrding, Note on continuous representations of Lie groups, Proc. Nat. Acad. Sci. 33 (1947), 331-332.

[Goo] R. Goodman, One-parameter groups generated by operators in an enveloping algebra, J. Funct. Anal. 6 (1970), 218-236.

[GW] R. Goodman and N. R. Wallach, Whittaker vectors and conical vectors, J. Funct. Anal. 39 (1980), 199-279.

[Nel] E. Nelson, Analytic vectors, Ann. of Math. 70 (1959), 572-615.

[Pou] N. S. Poulsen, On $C^{\infty}$-vectors and intertwining bilinear forms for representations of Lie groups, J. Funct. Anal. 9 (1972), 87-120.

[Rob] D W. Robinson, Lie groups and Lipschitz spaces, Duke Math. J. 57 (1988), 357-395.

[Tay] M. E. Taylor, Noncommutative harmonic analysis, Math. Surveys and Monographs, vol. 22, Amer. Math. Soc., Providence, RI, 1986.

[Wil] A. Wilansky, Modern methods in topological vector spaces, McGraw-Hill, New York, 1978.

Mathematics IAS, Australian National University, Canberra ACT 2601, Australia 